\title{
Gout of feet and ankles in different disease durations: diagnostic value of single-source DECT and evaluation of urate deposition with a novel semi-quantitative DECT scoring system
}

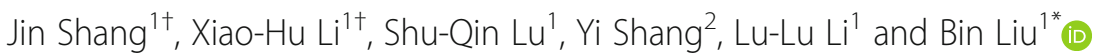

\begin{abstract}
Objectives: To investigate the diagnostic performance of single-source dual-energy computed tomography (DECT) based on gemstone spectral imaging technology (including Discovery CT750HD and Revolution CT) in patients with suspected feet/ankles gouty arthritis, and evaluate the urate deposition with a novel semi-quantitative DECT scoring system.

Methods: A total of 196 patients were consecutively included. Feet and ankles were evaluated in all patients by single-source DECT scan. The 2015 EULAR/ACR criteria were used as the reference for the diagnosis of gout. The sensitivity, specificity, and area under the receiver operating characteristic curve (AUC) of DECT for the diagnosis of gout in the early ( $\leq 1$ year), middle (1-3 years), and late ( $>3$ years) disease durations were calculated. Besides, a novel semi-quantitative DECT scoring system was assessed for the measurement of urate deposition, and the correlation between the scores and the clinical and serological data were also evaluated. Moreover, the influences of artifacts on the diagnostic performance of DECT were also determined.

Results: The sensitivity, specificity, and AUC of DECT in 196 patients were $38.10,96.43 \%$, and 0.673 in the earlystage group; $62.96,100.00 \%$, and 0.815 in the middle-stage group; and 77.55, 87.50\%, and 0.825 in the late-stage group, respectively. The overall diagnostic accuracies in the AUC of DECT (Discovery CT750HD and Revolution CT) in the middle and late stages of gout were higher than that in the early stage of gout. Besides, the monosodium urate crystals were deposited on the first metatarsophalangeal joints and ankles/midfeet. Age, the presence of tophus, bone erosion, and disease duration considerably affected the total urate score. No statistical difference in the positive detection of nail artifact, skin artifact, vascular calcification, and noise artifact was found between the case and control groups.
\end{abstract}

\footnotetext{
* Correspondence: Ibhyz32@163.com

${ }^{\dagger}$ Jin Shang and Xiao-Hu Li contributed equally to this work.

'Department of Radiology, The First Affiliated Hospital of Anhui Medical

University, 210 Jixi Road, Hefei 230022, Anhui, China

Full list of author information is available at the end of the article
}

(C) The Author(s). 2021 Open Access This article is licensed under a Creative Commons Attribution 4.0 International License, which permits use, sharing, adaptation, distribution and reproduction in any medium or format, as long as you give appropriate credit to the original author(s) and the source, provide a link to the Creative Commons licence, and indicate if changes were made. The images or other third party material in this article are included in the article's Creative Commons licence, unless indicated otherwise in a credit line to the material. If material is not included in the article's Creative Commons licence and your intended use is not permitted by statutory regulation or exceeds the permitted use, you will need to obtain permission directly from the copyright holder. To view a copy of this licence, visit http://creativecommons.org/licenses/by/4.0/. 
Conclusion: DECT (Discovery CT750HD and Revolution CT) showed promising diagnostic accuracy for the detection of urate crystal deposition in gout but had limited diagnostic sensitivity for short-stage gout. Longer disease duration, the presence of tophus, and bone erosion were associated with the urate crystal score system. The artifacts do not remarkably affect the diagnostic performance of DECT in gout.

Keywords: Gout, Dual-energy spectral CT, Scoring method, Disease duration, Artifact, Clinical trial

\section{Introduction}

Gout is the most common form of inflammatory arthropathy resulting from monosodium urate (MSU) crystals deposition [1]. The long-term deposition of MSU crystals in the joints and periarticular soft tissues can contribute to progressive joint destruction, renal failure, and mortality given its association with coronary heart disease, metabolic syndrome, and diabetes $[2,3]$. Early diagnosis and the timely initiation of therapy are imperative to improve the disease outcome of patients with gout. Gout diagnosis is based on the identification of negative birefringent MSU crystals in the joint fluid or tophi by polarized microscopy. However, this method is seldom used in clinical practice owing to many limitations, such as invasiveness, lack of training, and microscopy unavailability [4]. Thus, the clinical utilization of dual-energy computer tomography (DECT) for gout diagnosis has dramatically increased over the past decade. Image acquisition is based on two different spectra (often 80 and $140 \mathrm{kV}$ ) in DECT scanning that can operate at the source or detector level. Dual sourcedual detector (DECT scanners with two X-ray tubes), single-source rapid kilovoltage switching, twin-beam single-source CT with gold filter, and dual-scan singlesource are the most commonly used DECT technologies [5]. The validity of DECT as a measure for gout diagnosis has been confirmed in many previously published literatures [6-9]. Currently, DECT has been incorporated into the 2015 American College of Rheumatology/European League Against Rheumatism gout classification criteria (ACR /EULAR 2015) based on reliable previously published data and its diagnostic accuracy [10].

Most studies have shown that DECT is a highly accurate tool for gout diagnosis, but it has limited diagnostic accuracy for gout in short-term duration with widely varied results. Gamala et al. [11] conducted a metaanalysis of 10 studies on the diagnostic accuracy of DECT and revealed that the pooled sensitivity and specificity of DECT are 81 and $91 \%$, respectively, whereas the pooled sensitivity is $55 \%$ in patients with recent onset disease ( $\leq 6$ weeks). In a recent study of 15 patients with aspiration-proven gout with a disease duration of within 1 year, the sensitivity of DECT in identifying MSU deposition was only $26.6 \%$ [12]. Conversely, in a study of 40 patients in the early course of gout, the sensitivity and specificity of DECT for the diagnosis of gout are 90 and $83 \%$, respectively [8]. However, currently most studies on gout were performed using dual-source DECT $[8,12-14]$. and the performances of other types of DECT, such as single-source rapid kilovoltage switching DECT, and dual-scan single-source CT, were less studied. Thus, the present study was conducted using single-source rapid kilovoltage switching DECT based on gemstone spectral imaging (GSI) technology (including Discovery CT750HD and Revolution CT, GE Healthcare, Milwaukee, WI, USA), in which the single tube was switched between 80 and $140 \mathrm{kVp}$ in less than $0.05 \mu \mathrm{s}$.

Moreover, DECT has a potential role in evaluating MSU crystals deposition in patients with gout receiving urate-lowering treatments. At present, urate deposition is assessed by automated volume assessment software, but this time-consuming method could not easily measure the volume of specific deposition sites. Therefore, Bayat et al. [15] developed a semi-quantitative DECT scoring system in a large sample set that allowed the evaluation of urate deposition at specific sites of feet/ankles. In this scoring system, each scan is divided into four areas, and each area is graded in accordance with the maximum amount of urate deposition measured during visual examination. They found that the DECT urate scores are highly correlated with urate volumes and can differentiate between responders and nonresponders to intensive urate-lowering treatment. In the current study, we applied this semi-quantitative DECT scoring system for the evaluation of urate deposition in gout and assessed the correlation between the urate deposition scores evaluated by the DECT GSI at feet/ankles and the clinical data.

Furthermore, the artifacts produced during DECT scanning and post-processing may lead to false-positive results. However, the differentiation and clinical importance of artifacts in DECT for MSU deposition identification are still controversial. Chou et al. [3] summarized the common artifacts in DECT imaging for gout, such as nail bed artifact, skin artifact, clumpy artifacts along the tendon, beam hardening, patient motion during the scan, and urate-like pixelations in vascular calcification. Some scholars proposed that these artifacts are true artifacts $[6,16]$. Roddy et al. [17] and other scholars $[6,18]$ suggested that nail bed and tendon artifacts have important influence on gout diagnosis. However, whether this influence is due to true gouty deposition or an artifact is unknown. 
Thus, the aims of our study were:

- to evaluate the sensitivity, specificity, and accuracy of single-source DECT GSI (Discovery CT750HD and Revolution CT, GE Healthcare, Milwaukee, WI, USA) for the detection of MSU crystal deposition at feet/ ankles in patients with suspected gout in early $(\leq 1$ year), middle ( $1-3$ years), and late ( $>3$ years) stages;

- to determine the correlation between urate deposition evaluated by single-source DECT GSI at feet/ankles and clinical data;

- to investigate whether artifacts affect the diagnostic performance of single-source DECT in gout.

\section{Methods}

\section{Study patients}

Patients with suspected gout of the feet or ankles were consecutively included in the retrospective study from December 2016 to December 2020 in the hospital. The final gout diagnosis was determined by experienced rheumatologists using the 2015 EULAR/ACR classification criteria as the reference [10]. Characteristic data, including age, sex, disease duration, body mass index (BMI), serum uric acid (SUA) level at time of DECT (the interval between the test of SUA and performance of DECT within 3 days), urate-lowering treatments (ULT) at time of DECT (medication treatment on the day of DECT examination), Diuretic use at time of DECT, the presence of subcutaneous tophi, erosive disease, renal function described as eGFR, time from last gouty attack to DECT examination, and patient-reported concomitant disease (such as hypertension, ischemic heart disease, dyslipidemia, and type II diabetes), were obtained from the medical records.

Patients were selected using the following inclusion criteria: patients aged between 20 and 90 years old; patients undergoing two single-source DECT systems with same technique (Discovery CT750HD and Revolution CT, GE Healthcare, Milwaukee, WI, USA) of their feet and ankles; patients in the gout group receiving the final diagnosis using 2015 EULAR/ACR criteria after comprehensive musculoskeletal examinations; patients in the control with the final diagnosis of rheumatoid arthritis, inflammatory polyarthropathy, achilles tendinitis, and psoriatic arthritis. Exclusion criteria for patients were: relevant foot or ankle trauma, or surgery procedures. Moreover, there was no comparison with other imaging methods (ultrasonography, MRI, etc.).

Ethical approval was provided by the hospital ethics committee. The study was conducted according to the Helsinki Declaration.

\section{DECT examination}

All scans were performed using single-source DECT systems (Discovery CT750HD and Revolution CT). The tube voltage of the two systems was switched between 80 and $140 \mathrm{kVp}$ in less than $0.05 \mu \mathrm{s}$. In the Discovery CT750HD DECT system (GE Healthcare, Milwaukee, WI, USA), the pitch was 0.984 , the beam collimation was $40 \mathrm{~mm}$, the gantry rotation time was $0.6 \mathrm{~s}$, the slice thickness was $5 \mathrm{~mm}$, and the reconstruction increment was $1.25 \mathrm{~mm}$. In the Revolution CT system (GE Healthcare, Milwaukee, WI, USA), the pitch was 0.992, the beam collimation was $80 \mathrm{~mm}$, the gantry rotation time was $0.8 \mathrm{~s}$, the slice thickness was $5 \mathrm{~mm}$, and the reconstruction increment was $1.25 \mathrm{~mm}$. Both feet were scanned simultaneously in supine position without an intravenous contrast agent.

DECT GSI translated two sets of absorption projection data to material density projection data and provided different base material mapping (such as uric acid/calcium pairing, water/iodine pairing, water/calcium pairing, etc.). Base material concentration imaging can better reflect the content of the corresponding components of the tissue, and the uric acid crystal can be visualized more accurately in the uric acid-based map than that in the calcium-based map [19].

\section{Image analysis}

Two musculoskeletal radiologists blinded to the patients' information independently reviewed the images for the detection and localization of MSU deposits. Each site was deemed positive or negative for the presence of MSU crystals. One positive presence in a single joint was enough to count the patient as having gout. Four regions were involved in the scoring system: the first metatarsophalangeal $\left(\mathrm{MTP}^{\text {st }}\right)$ joints, other joints of the toes (2nd-5th MTP and interphalangeal joints), ankles/ midfeet, and tendons. Each joint was scored as follows: $0=$ no deposit, $1=$ dots, $2=$ single deposit, $3=$ more than one deposit. The presence of artifacts at the feet/ankles was also recorded. If discordant results arose after the consultation of the two readers, the two reviewer and a third co-author with more than 30 years of experience in musculoskeletal radiology jointly re-examined the controversial images to achieve a consensus that would be used for final analyses described in this current study.

\section{Statistical analyses}

The SPSS software version 26.0 (SPSS Inc., Chicago, IL, USA) and MedCalc statistical software version 19.2.6 (MedCalc Software bvba, Ostend, Belgium) were used for statistical analyses. Sensitivity, specificity, and area under receiver operating characteristic curve (AUC) were calculated. Continuous data were described as means and standard deviation (SD), while categorical data were expressed as frequencies and percentages. The statistical significance for the differences between quantitative variables without normal distribution were calculated 
by Mann-Whitney test, whereas for those with normal distribution we used the Student's t-test. The inter-reader reliability analyses for the diagnostic performance of DECT were assessed using Cohen's kappa statistic that was interpreted as follows: the values of $0.00-0.20$ representing as "slight" reliability; $0.21-0.40$ as "fair" reliability; $0.41-0.60$ as "moderate" reliability; 0.61-0.80 as "substantial" reliability; and $>0.80$ as "almost perfect" reliability. Cramer's V was utilized to correlate the DECT findings that indicate the presence of MSU crystal deposits at feet/ankles with the clinical and serological variables. Statistical significance was evaluated by Chi-square and Fisher's exact test. $P<0.05$ was regarded as the statistical significance.

\section{Results}

\section{Clinical characteristic}

An overall number of 196 patients were recruited in the study. The demographic characteristics of the patients are shown in Table 1. Among the included patients, 125 (mean age, $56.16 \pm 16.93$ years) were examined using the Discovery CT750HD, whereas 71 (mean age, $51.68 \pm$ 17.59 years) were examined using the Revolution CT. Mean disease duration was $7.52 \pm 7.94$ years. A history of tophus was found in $16.8 \%(33 / 196)$ of patients, and the mean uric acid level at recruitment was $456.11 \pm 123.16$ $\mathrm{mmol} / \mathrm{L}$. Thirty-seven patients were receiving uratelowering treatment during evaluation. There were significant differences in disease duration, presence of tophus, and diuretic use between the gout and control groups $(P<0.05)$, and the related outcomes should be interpreted cautiously. Meanwhile, subgroup analysis on disease duration was performed regarding the early-, middle-, and late- stages to reduce the bias. Moreover, we recorded positive DECT results (Fig. 1) and scored the urate deposition in four locations (MTP1st joints, other joints of the toes, ankles/midfeet, and tendons).

\section{Diagnostic performance of single-source DECT GSI in different stages of gout}

The sensitivity, specificity, and AUC of single-source DECT GSI in 196 patients for the diagnosis of gout were $38.10,96.43 \%$, and 0.673 in the early-stage group; 62.96 , $100.00 \%$, and 0.815 in the middle-stage group; 77.55 , $87.50 \%$, and 0.825 in the late-stage group, and 69.18 , $94.00 \%$, and 0.816 in the total-stages group, respectively (Table 2 and Fig. 2). The sensitivity, specificity, and AUC of gout diagnosis using Discovery CT750HD $(n=$ 125) were $55.56,100.00 \%$, and 0.778 in the early-stage group; $59.09,100.00 \%$, and 0.795 in the middle-stage group; $75.71,85.71 \%$, and 0.807 in the late-stage group, and $70.30,95.83 \%$, and 0.831 in the total-stages group, respectively (Table 2 and Additional files 1 ). The sensitivity, specificity, and AUC in gout diagnosis using Revolution CT $(n=71)$ were $25.00,93.33 \%$, and 0.592 in the early-stage group; $80.00,100.00 \%$, and 0.900 in the middle-stage group; $82.14,88.89 \%$, and 0.855 in the latestage group, and $66.67,92.31 \%$, and 0.795 in the totalstages group, respectively (Table 2 and Additional files 2). The overall diagnostic accuracies of AUC in DECT GSI (Discovery CT750HD and Revolution CT) in the late and middle stages of gout were higher than that in the early stage of gout. Moreover, the cross-tabulations of diagnostic performance of DECT based on different disease durations are shown in Additional files 3, 4, 5, and 6.

\section{The inter-reader reliability analyses for the diagnostic performance}

The values for inter-reader agreements of Discovery CT, Revolution CT, and both CT scanners were 0.879 , 0.867 , and 0.874 in the early stage; $0.923,0.720$, and 0.879 in the middle stage; and $0.853,0.885$, and 0.864 in the late stage. The strength of agreements was considered good or very good.

\section{Measurement of urate deposition using the semi- quantitative DECT scoring system}

The MSU crystals were deposited on MTP1st joints $(1.43 \pm 2.06$ scores $)$ and ankles/midfeet (1.44 \pm 2.32 scores), followed by tendons $(0.71 \pm 1.68$ scores $)$ and other joints of the toes $(0.54 \pm 1.46$ scores $)$. The scores were significantly higher in patients with a disease duration of more than 3 years than those with a duration of less than 3 years (Table 3 ).

\section{Associations between total urate deposit score and gout characteristics}

Age, presence of tophus, bone erosion, and disease duration significantly affected the total urate score $(P<$ 0.050), whereas gender, SUA level at time DECT, uratelowering treatments, BMI, time from last gouty attack to DECT exam, and renal function did not significantly affect the total urate score $(P>0.050$, Table 4$)$.

\section{Artifacts in gout and non-gout groups}

Four types of artifacts, namely, nail artifact, skin artifact, noise artifact, and vascular calcification, were found in the current study (Fig. 3). No statistical difference in the detection of these four types of artifacts including nail artifact $(P=0.143)$, skin artifact $(P=0.307)$, vascular calcification $(P=0.341)$, and noise artifact $(P=1.000)$ was found between the case and control groups (Table 5). Therefore, artifacts do not remarkably affect the diagnostic performance of DECT in gout.

\section{Discussion}

Gout is caused by the deposition of MSU crystals in the joints or surrounding tissues, among which, the MTP1 $1^{\text {st }}$ joint is the most common joint involved in gout [20]. 


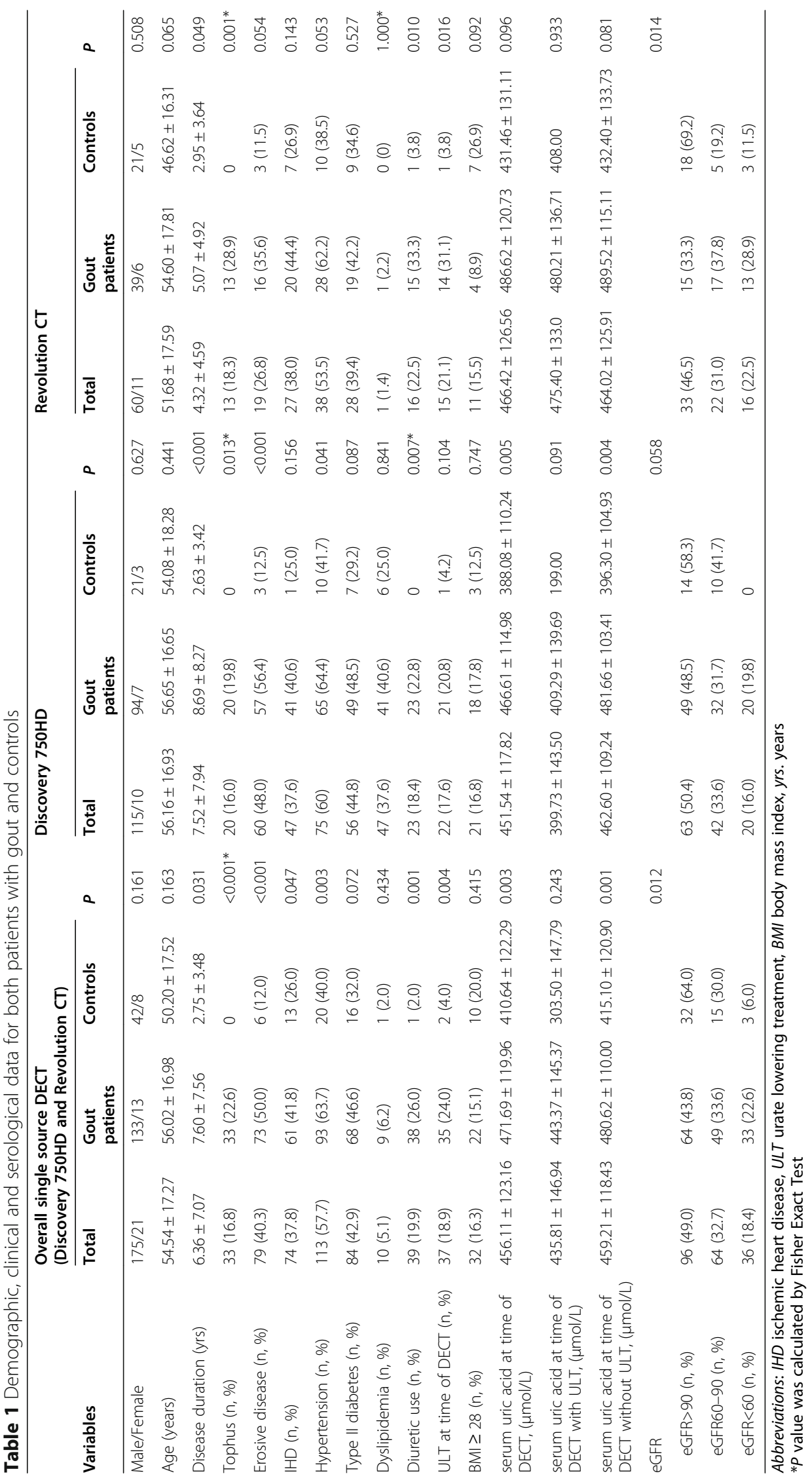



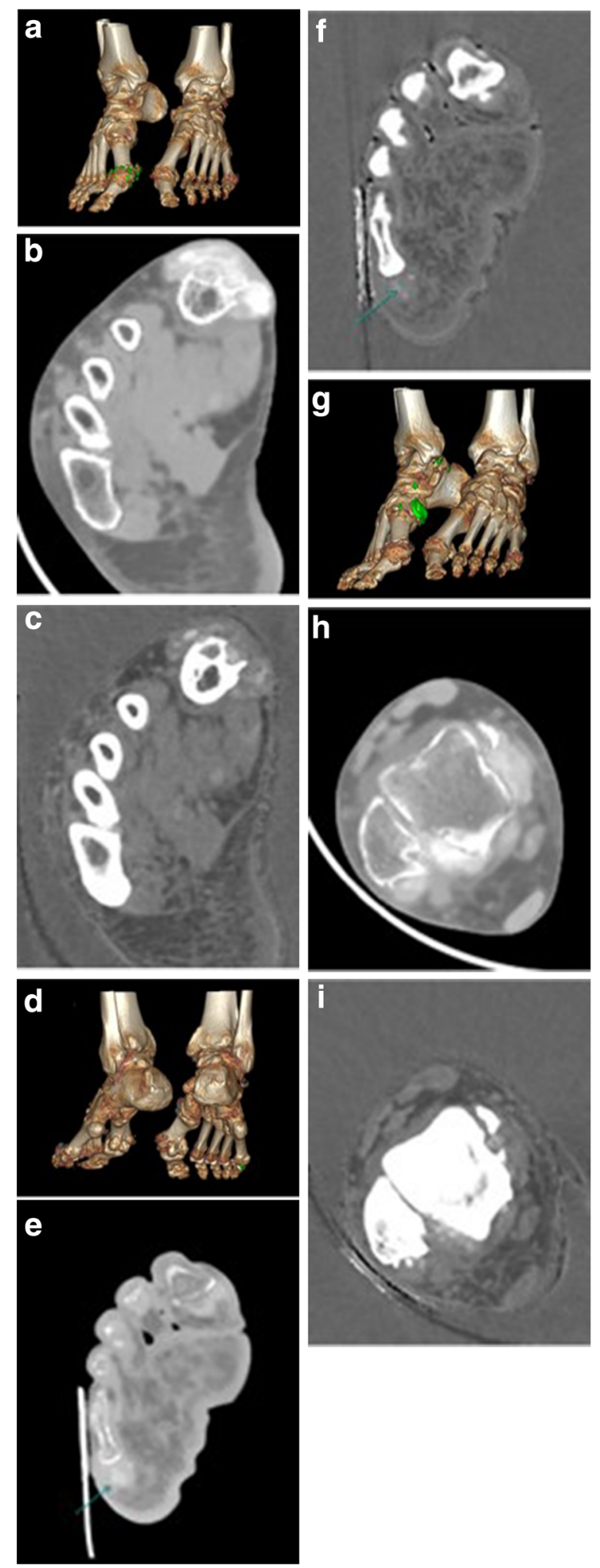

Fig. $13 \mathrm{D}$ reconstruction (a), uric acid base image $(\mathbf{b})$, calcium base image (c) of the same patient showed the identification of score 3 for MTP1 $^{\text {st }}$ in the foot. (d), uric acid base image (e), calcium base image ( $\mathbf{f}$ ) of the same patient showed the identification of score 2 for other joints of the toes in the foot. (g), uric acid base image (h), calcium base image (i) of the same patient showed the identification of score 3 for $M \mathrm{MTP}^{1}{ }^{\text {st }}$ in the foot for ankle/midfoot in the foot

The incidence of gout has been increasing annually with the improvement of living standards and changes in diet [6]. Long-term urate deposition is closely related to diabetes mellitus, coronary heart disease, metabolic disorder, and other diseases, which result in serious complications, including joint destruction [21]. Therefore, the early diagnosis and timely treatment of gout are important for the prognosis of patients with gout. Most of the experimental studies on urate deposition were conducted using dual-source devices [12-14, 22]. The current study used single-source systems (DECT GSI: Discovery CT750HD and Revolution CT) to explore the diagnostic performance of DECT in patients with different disease durations and correlate urate deposition score with clinical characteristics. Furthermore, whether artifacts have impacts on gout diagnosis was also investigated.

Dual-source DECT has been reported as a highly accurate tool for gout diagnosis $[6,7,9]$. However, the studies regarding the diagnostic performance of singlesource DECT were rare. Glazebrook et al. [6] conducted a retrospective study on 12 patients with aspirationproven gout, and reported that the sensitivity and specificity of dual-source DECT are 100 and 89\%, respectively. Jia et al. [14] concluded that the sensitivity of dual-source DECT is lower by $35.7 \%$ in patients with onset gout. Zhang et al. [12] compared the diagnostic accuracies of dual-source DECT and ultrasonography in patients with different gouty disease durations and reported that the sensitivities of DECT for gout within 1 year, 1-3 years, and more than 3 years are 26.6, 66.6, and $90 \%$, respectively. They also revealed that the sensitivity of ultrasonography is remarkably higher than that of dual-source DECT in early gout and suggested ultrasonography as the first choice for the diagnosis of earlystage gout. By contrast, Bongartz et al. [8] reported a higher sensitivity of $80 \%$ using dual-source DECT in 20 gout patients with symptom duration of $<6$ weeks. In the present study, the sensitivities of single-source DECT in middle- and late-stage gout were remarkably higher than that in early-stage gout. The outcomes demonstrated that disease duration strongly affects the diagnostic accuracy of single-source DECT, and DECT has limited diagnostic value in early-stage gout.

The results could be explained as follows. First, DECT could only detect the smallest size of $2 \mathrm{~mm}$ in diameter 


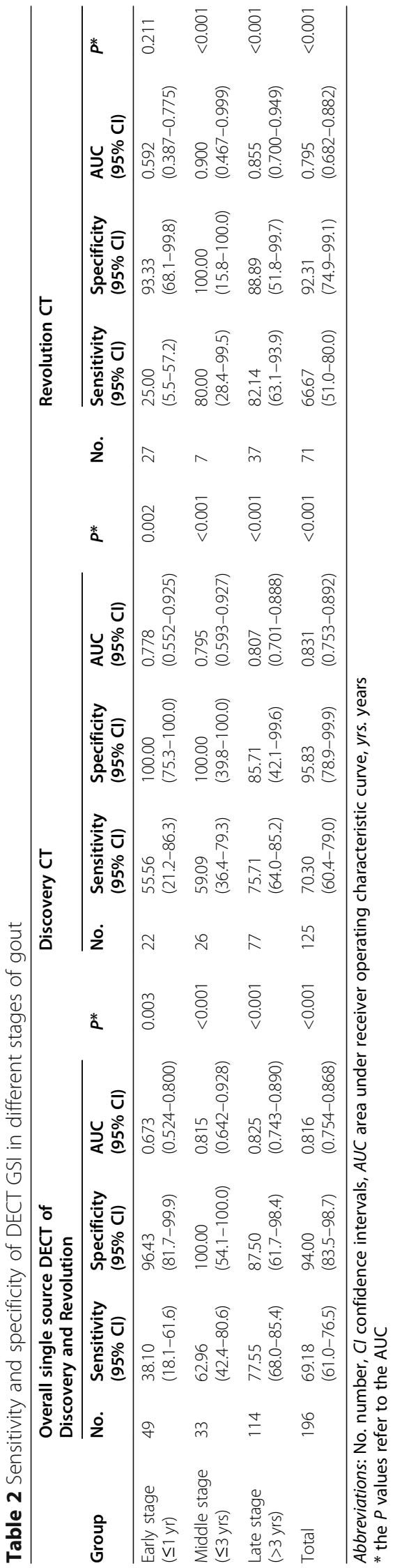


a

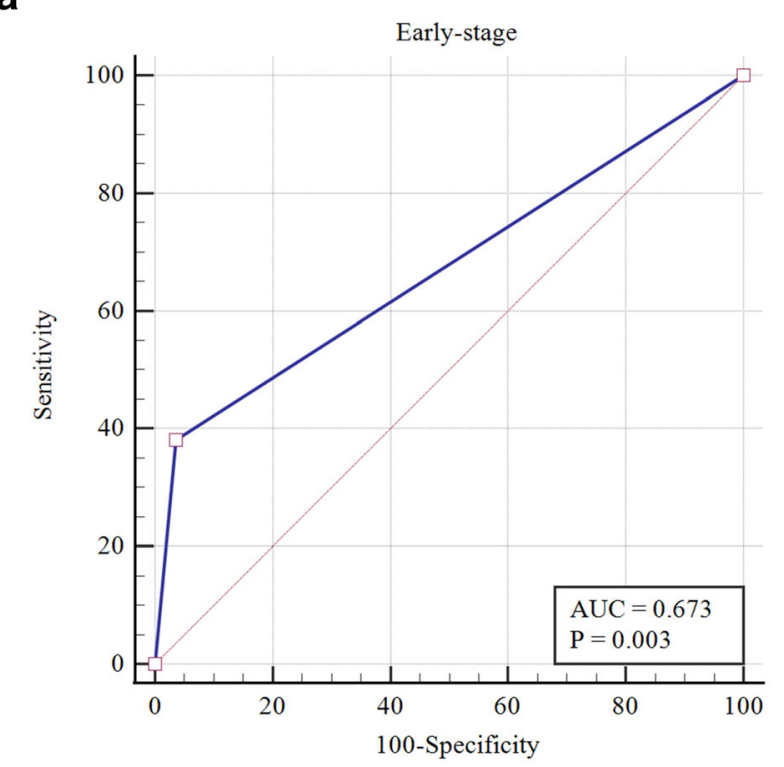

b

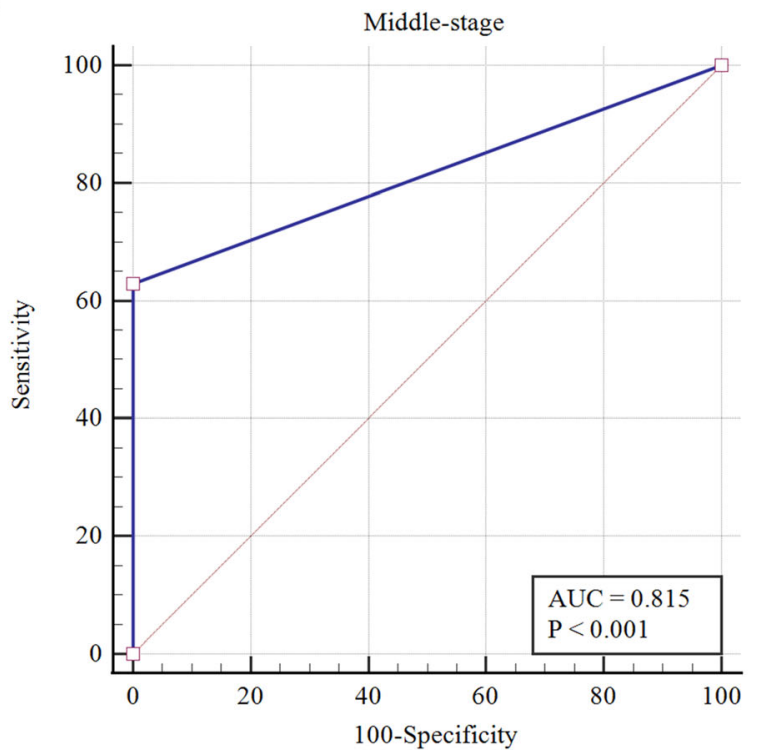

C

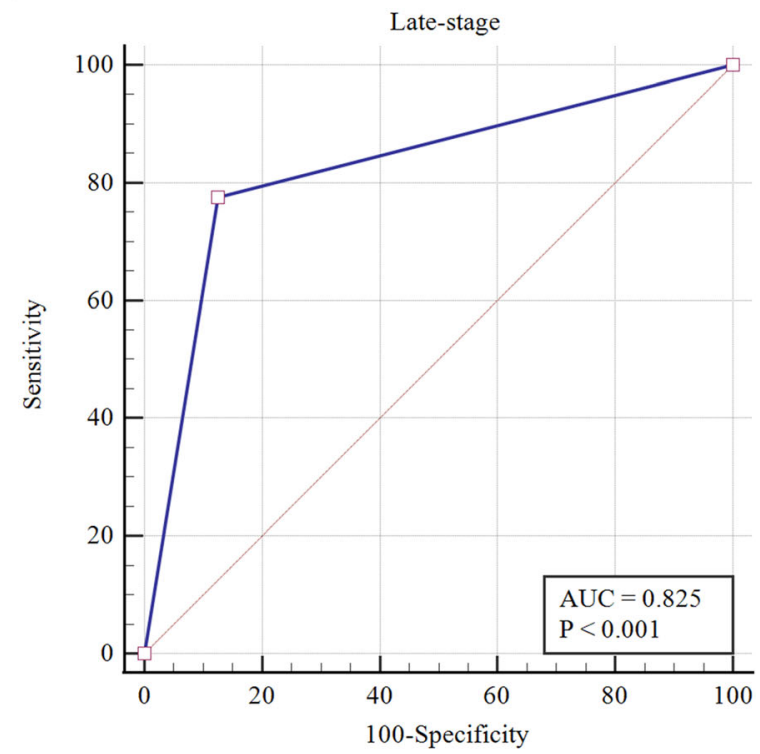

d

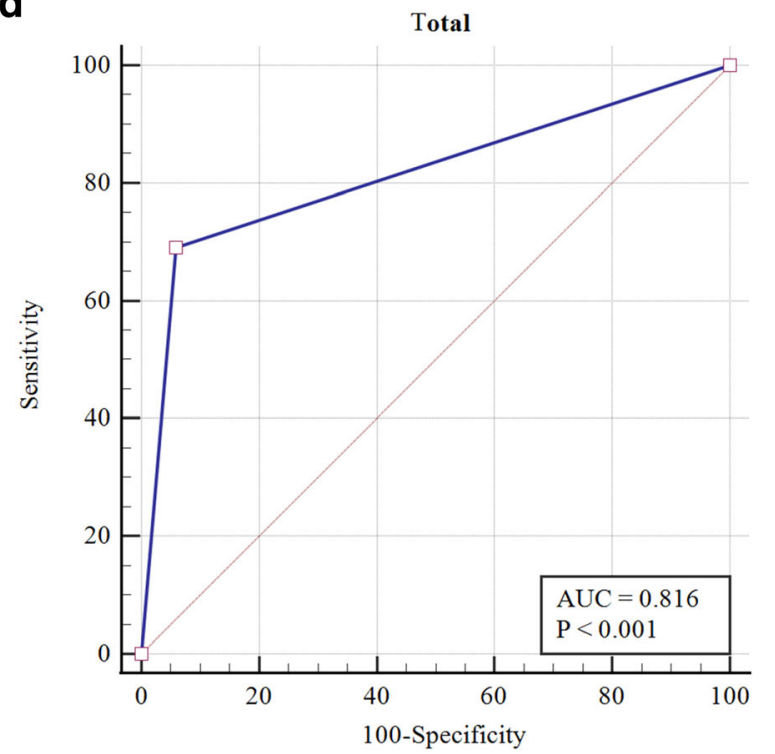

Fig. 2 a ROC curve for sensitivity, 1-specificity, and AUC regarding the early-stage of gout with overall single source DECT Discovery and Revolution. b ROC curve for sensitivity, 1-specificity, and AUC regarding the middle-stage of gout with overall single source DECT Discovery and Revolution. c ROC curve for sensitivity, 1-specificity, and AUC regarding the late-stage of gout with overall single source Discovery CT750 HD and Revolution CT. d ROC curve for sensitivity, 1-specificity, and AUC regarding the total-stages of gout with overall single source Discovery CT750 HD and Revolution CT

and a minimum volume concentration of $15-20 \%$ $[16,23]$. However, the volume of MSU crystals in patients with short disease course was small and undetected. Thus, the sensitivity of single-source DECT is decreased in the early stages of gout. Wu et al. [9] and Jia et al. [14] found a strong relationship between disease duration and MSU crystal volume, which can partly explain the higher diagnostic effect of DECT in patients with late-stage gout. Most studies on gout presented a relatively long disease duration, and the overall high diagnostic accuracy is largely attributed to the high MSU crystal volume in the late stage of gout $[12,14,24]$. Moreover, the deposition of MSU crystals in the early stage of gout is also affected by the active chemotaxis and phagocytosis of leukocytes. The detection of single-source DECT for MSU crystals might be adversely affected by these chemical compositions [22]. Future researches should test the reliability of single-source DECT in early gout and find an optimal imaging approach for the identification of MSU deposits in early-stage disease. 


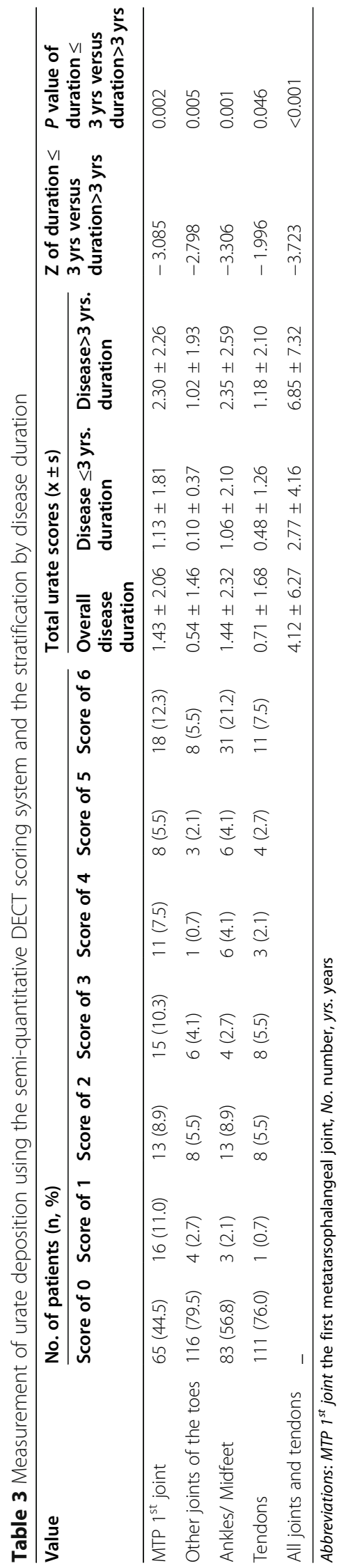


Table 4 Associations between total urate deposit score and gout characteristics

\begin{tabular}{|c|c|c|c|c|}
\hline Factors & $\mathrm{n}$ & Total urate deposit score & $t / Z / F^{a}$ & $P$-value \\
\hline Age (years) & & & -2.040 & 0.041 \\
\hline$<56$ & 63 & $4.27 \pm 5.78$ & & \\
\hline$\geq 56$ & 83 & $6.45 \pm 7.25$ & & \\
\hline Gender & & & -0.91 & 0.928 \\
\hline Men & 133 & $5.62 \pm 6.87$ & & \\
\hline Women & 13 & $4.38 \pm 5.06$ & & \\
\hline Tophus & & & -6.157 & $<0.001$ \\
\hline Yes & 33 & $12.45 \pm 7.65$ & & \\
\hline No & 113 & $3.48 \pm 4.84$ & & \\
\hline Erosive disease & & & -6.188 & $<0.001$ \\
\hline Yes & 73 & $8.55 \pm 7.52$ & & \\
\hline No & 73 & $2.47 \pm 3.96$ & & \\
\hline Disease duration (yrs) & & & -3.723 & $<0.001$ \\
\hline$\leq 3$ & 48 & $2.77 \pm 4.16$ & & \\
\hline$>3$ & 98 & $6.85 \pm 7.32$ & & \\
\hline Urate at DECT $(\mu \mathrm{mol} / \mathrm{L})$ & & & -1.752 & 0.080 \\
\hline$\leq 585$ & 26 & $8.12 \pm 7.90$ & & \\
\hline$>585$ & 120 & $4.94 \pm 6.33$ & & \\
\hline ULT use at DECT & & & -1.528 & 0.127 \\
\hline Yes & 35 & $7.20 \pm 7.88$ & & \\
\hline No & 111 & $4.97 \pm 6.26$ & & \\
\hline BMI $\left(\mathrm{kg} / \mathrm{m}^{2}\right)$ & & & -0.756 & 0.450 \\
\hline$<28$ & 124 & $5.53 \pm 6.56$ & & \\
\hline$\geq 28$ & 22 & $5.36 \pm 7.73$ & & \\
\hline $\begin{array}{l}\text { Time from last gouty attack to } \\
\text { DECT examination (day) }\end{array}$ & & & -0.883 & 0.405 \\
\hline$\leq 10$ & 66 & $4.94 \pm 6.90$ & & \\
\hline$>10$ & 66 & $5.45 \pm 6.84$ & & \\
\hline Renal function by eGFR & & & 0.981 & 0.378 \\
\hline$\geq 90$ & 64 & $4.62 \pm 6.06$ & & \\
\hline $60-90$ & 49 & $6.18 \pm 6.77$ & & \\
\hline$<60$ & 33 & $6.21 \pm 7.81$ & & \\
\hline
\end{tabular}

${ }^{a}$ The $\mathrm{t}$ value, $\mathrm{Z}$ value, and $\mathrm{F}$ value were obtained by Student's $\mathrm{t}$-test, Mann-Whitney test, and Analysis of variance, respectively, according to the result of the test for normal distribution

Besides, growing evidence shows that DECT can determine disease activity and therapy efficacy in gout [25]. Bayat et al. [15] developed a semi-quantitative DECT scoring method for the evaluation of MSU crystal deposits at specific sites in the feet/ankles during therapy, and the scores were highly correlated with urate volumes. Dalbeth et al. [26] measured the MSU crystal deposition in patients with gout who received stabledose allopurinol and demonstrated that the higher crystal deposition on DECT was associated with higher SUA and lower allopurinol dose. However, the results of our study showed that SUA level at time of DECT. are not correlated with the total urate deposit score, which might be attributed to the fact that for some patients with gout, the SUA levels are in the normal range, or even lower [27]. Urano et al. [27] concluded that the decrease in SUA during acute gouty arthritis was associated with increased urinary excretion of uric acid. In addition, we also found that there was no correlation of use of urate-lowering treatment with the total urate deposit score. Dehlin et al. [28] showed the suboptimal treatment using urate-lowering treatment in gout and suggested that the efficiencies of urate-lowering treatment with long-term periods were limited for patients 

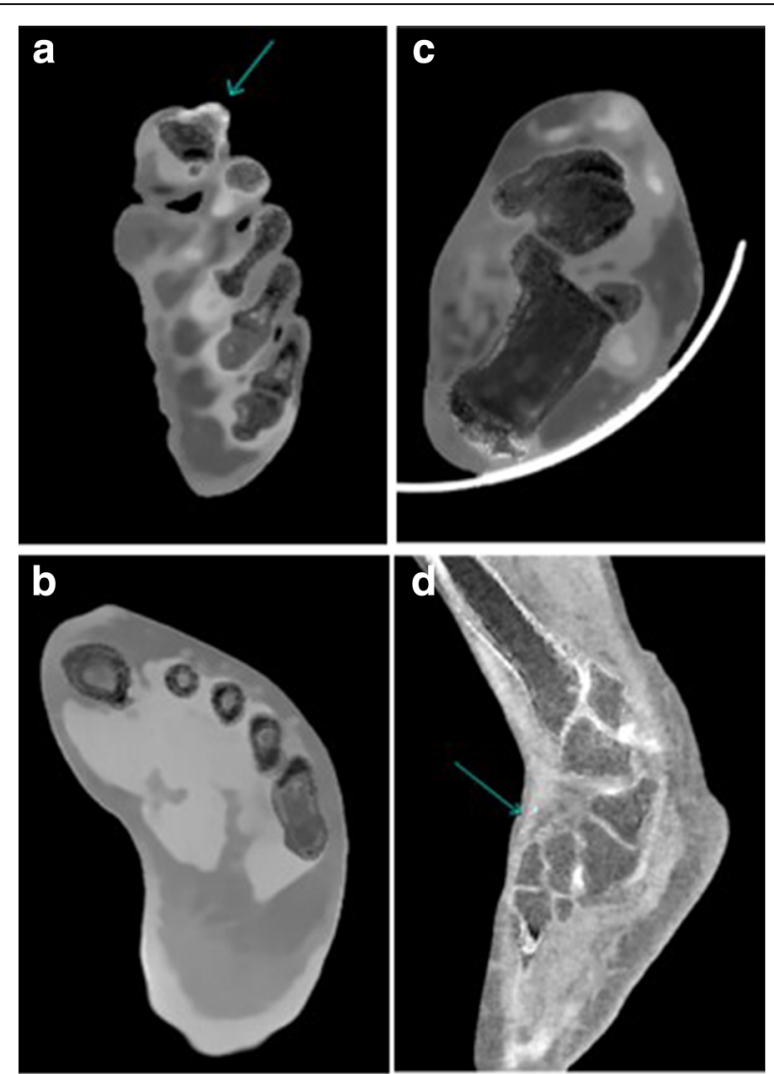

Fig. 3 a Uric acid base image (a) depicted typical artifact from the nail bed. b Uric acid base image (b) depicted typical artifact from the skin. c Uric acid base image (c) depicted typical artifact from the noise. $\mathbf{d}$ Uric acid base image (d) depicted typical artifact from the vascular calcification

who had difficulties in compliance to the advice of doctor, thereby contributing to a health care problem of urate-lowering treatment management. Moreover, welltreated patients are slow "MSU deposit depletors" and still have substantial urate volumes even after 2 years. Patients unevenly reduce their MSU burden after uratelowering treatment. Thus, adding a density measurement of MSU crystal deposition to the apparent volume assessment may help understand the varying kinetics of MSU burden depletion [29, 30]. In addition, some of the patients enrolled in our study used urate-lowering treatment for a short time after gout diagnosis. These factors above could explain the unrelatedness of uratelowering treatment use and the total urate deposit scores. Furthermore, the results of our study also showed that the total urate deposit score was higher in patients with longer disease duration and correlated strongly to the presence of tophus, bone erosion, and disease duration. Similarly, Svensson et al. [31] applied this urate scoring method and found that the amount of MSU deposits is associated with the presence of tophus and disease duration. Dalbeth et al. [26] found that higher urate deposits are correlated with tophi and bone erosion. The reason why the presence of tophus, bone erosion, and longer disease duration caused higher amount of urate deposition could be explained by the following reasons. First, disease duration is the major contributor to urate deposition; urate deposition increases with disease duration $[9,14]$. Second, the presence of tophus is a dominant factor for bone erosion in gout [32]. Moreover, the presence of tophus and bone erosion result in a longer disease duration in patients with gout.

There are differences between single- and dual-source DECT. As for the dual-source DECT, two tube-detector pairs are employed and the tube voltages can be adjustable with the advantage of fast single energy combinations. However, the two separate tubes are offset by approximately $90^{\circ}$ to each other, thereby contributing to the material decomposition that is required to be performed only on the image domain because of the spatial offset between acquisitions [33]. In terms of the singlesource rapid kilovoltage switching scanners (Discovery CT750 HD and Revolution CT), there are almost notemporal mismatch and full feilds of view with the $\mathrm{X}$-ray tube switching between 80 and $140 \mathrm{kVp}$ in less than 0.2 ms. [34] Thus, multiple spectral images are generated by projection-space decomposition. As opposed to dualsource DECT systems, projection-space decomposition has the advantages of greater flexibility in the types of materials that can be used of data to minimize beam hardening artifacts [33].

DECT has limitations in gout diagnosis. First, the artifacts are commonly observed in the feet and ankles and

Table 5 Presence of artifacts in both patients with gout and controls

\begin{tabular}{|c|c|c|c|c|c|c|c|c|c|}
\hline \multirow[t]{2}{*}{ Group } & & \multicolumn{2}{|c|}{ Nailartifact } & \multicolumn{2}{|c|}{ Skinartifact } & \multicolumn{2}{|c|}{ Noise } & \multicolumn{2}{|c|}{ Vascular calcification } \\
\hline & & + & - & + & - & + & - & + & - \\
\hline Gout patients & 146 & 67 & 79 & 30 & 116 & 6 & 140 & 1 & 145 \\
\hline Controls & 50 & 17 & 31 & 7 & 43 & 0 & 50 & 0 & 50 \\
\hline Cramer's V & & 0.105 & & 0.073 & & 0.1 & & 0.042 & \\
\hline$x^{2}$ & & 2.150 & & 1.043 & & - & & - & \\
\hline$P$-value & & 0.143 & & 0.307 & & 0.3 & & $1.000^{*}$ & \\
\hline
\end{tabular}

${ }^{*} P$ value was calculated by Fisher Exact Test 
might interfere with radiologists' performance and experience. Some scholars believed that tiny scattered green pixelation within tendons may be the result of the subclinical deposition of MSU crystals [6, 18]. Besides, Chen et al. [35] demonstrated that nail urate could be a proxy for the burden of MSU deposition. We found no statistical difference in the positive detection of nail artifact, skin artifact, vascular calcification, and noise artifact between the case and control groups. Furthermore, the artifacts caused by noise and motion were not seen in the present study, which is probably attributed to the ultrafast reconstruction algorithms by the scintillator of the DECT GSI equipment with fast sampling capabilities $(\sim 50 \mu \mathrm{s})$ [36]. Moreover, the ionizing radiation of DECT is harmful to patients, although the radiation doses of DECT are comparable to or even lower than the dose reference level of body CT [37]. In addition, DECT has been described as a highly accurate tool for the detection and measurement of disease burden and is thus well-suited to evaluate the treatment response of gout. However, the presence of MSU deposits is not necessarily associated with gout, although they increase the risk of its occurrence. Meanwhile, DECT cannot be utilized for the establishment of inflammation. Thus, DECT can diagnose these deposits that contribute to the diagnosis of gout instead of directly diagnosing gout. In the criteria of ACR /EULAR 2015, DECT is one of the criteria, not the only and sufficient one. Moreover, DECT is not widely available and only restricted to certain radiology centers. Nevertheless, DECT provides material characterization via two or more X-ray photon energy-dependent attenuation, which allows the qualitative and quantitative determination of MSU deposition in joints and tissues. Furthermore, DECT performs an excellent visualization of deeper or complex structures and display the anatomic extent of gouty deposits.

This study has several limitations. First, this study was a cross-sectional study and therefore cannot determine the relationship between changes in urate deposition and urate-lowering treatment. Second, no reliable information on the characteristics of gout attack, such as the severity and frequency of attacks, was obtained in the medical records. Third, we did not compare our method with other types of DECT techniques to determine any difference in gout diagnosis. Finally, a few cases were confirmed by arthrocentesis, which is the gold standard for gout diagnosis. Alternatively, we utilized the 2015 EULAR/ACR classification criteria as reference instead of the invasive method.

\section{Conclusion}

DECT GSI (Discovery CT750HD and Revolution CT) showed promising diagnostic accuracy for the detection of MSU crystal deposition in gout but had limited diagnostic sensitivity for short-stage gout. Longer disease duration, the presence of tophus, and bone erosion were associated with the novel semi-quantitative DECT scoring system. Artifacts do not remarkably affect the diagnostic performance of DECT in gout.

\section{Supplementary Information}

The online version contains supplementary material available at https://doi. org/10.1186/s42358-021-00194-4

Additional file 1. a. ROC curve for sensitivity, 1-specificity, and AUC regarding the early-stage of gout with Discovery CT750 HD. b. ROC curve for sensitivity, 1-specificity, and AUC regarding the middle-stage of gout with Discovery CT750 HD. c. ROC curve for sensitivity, 1-specificity, and AUC regarding the late-stage of gout with Discovery CT750 HD. d. ROC curve for sensitivity, 1-specificity, and AUC regarding the total-stages of gout with Discovery CT750 HD.

Additional file 2. a. ROC curve for sensitivity, 1-specificity, and AUC regarding the early-stage of gout with Revolution CT. b. ROC curve for sensitivity, 1-specificity, and AUC regarding the middle-stage of gout with Revolution CT. c. ROC curve for sensitivity, 1-specificity, and AUC regarding the late-stage of gout with Revolution CT. d. ROC curve for sensitivity, 1 specificity, and AUC regarding the total-stages of gout with Revolution CT.

Additional file 3. The cross-tabulation of diagnostic performance of DECT in the early stage ( $\leq 1 \mathrm{yrs}$ ).

Additional file 4. The cross-tabulation of diagnostic performance of DECT in the middle stage ( $\leq 3 \mathrm{yrs}$ ).

Additional file 5. The cross-tabulation of diagnostic performance of DECT in the late stage ( $>3$ yrs)

Additional file $\mathbf{6}$. The cross-tabulation of diagnostic performance of $\mathrm{DECT}$ in all the disease durations.

\section{Acknowledgements}

The authors thank Lu-Ping Zhou for his invaluable assistance in the editing of this manuscript.

\section{Authors' contributions}

Jin Shang: Conceptualization, Data curation, Formal analysis, Investigation, Methodology, Software, Validation, Visualization, Writing - original draft, Writing - editing. Xiao-hu Li: Conceptualization, Project administration, Data curation, Formal analysis, Methodology, Writing - review. Shu-qin Lu:

Conceptualization, Data curation, Formal analysis, Methodology, Supervision, Validation, Visualization, Writing - review. Yi Shang: Conceptualization, Data curation, Formal analysis, Methodology, Validation, Visualization, Writing review. Lu-lu Li: Data curation, Formal analysis, Methodology, Validation, Visualization, Writing - review. Bin Liu: Conceptualization, Project administration, Supervision, Validation, Writing - review. The author(s) read and approved the final manuscript.

\section{Authors' information}

Jin Shang: MD, Department of Radiology, the First Affiliated Hospital of Anhui Medical University, 210 Jixi Road, Hefei, Anhui 230022, China. Xiao-Hu Li: PhD, Department of Radiology, the First Affiliated Hospital of Anhui Medical University, 210 Jixi Road, Hefei, Anhui 230022, China. Shu-Qin Lu: MD, Department of Radiology, the First Affiliated Hospital of Anhui Medical University, 210 Jixi Road, Hefei, Anhui 230022, China. Yi Shang: MD, Outpatient Department of The Second Central Division, The General Hospital of PLA, 28 Fuxing Road, Beijing 100853, China.

Lu-Lu Li: MD, Department of Radiology, the First Affiliated Hospital of Anhui Medical University, 210 Jixi Road, Hefei, Anhui 230022, China.

Bin Liu: MD, Department of Radiology, the First Affiliated Hospital of Anhui Medical University, 210 Jixi Road, Hefei, Anhui 230022, China. 


\section{Availability of data and materials}

Not applicable.

\section{Declarations}

\section{Ethics approval and consent to participate}

Ethical approval was provided by the ethics committees of the First Affiliated Hospital of Anhui Medical University Ethics Committee. Since this is a retrospective study, formal consent is not required.

\section{Consent for publication}

Not applicable.

\section{Competing interests}

None of the authors have any competing interest.

\section{Author details}

'Department of Radiology, The First Affiliated Hospital of Anhui Medical University, 210 Jixi Road, Hefei 230022, Anhui, China. ${ }^{2}$ Outpatient Department of The Second Central Division, The General Hospital of PLA, Beijing 100853, China.

Received: 26 March 2021 Accepted: 2 June 2021

Published online: 12 June 2021

\section{References}

1. Kuo CF, Grainge MJ, Zhang W, Doherty M. Global epidemiology of gout: prevalence, incidence and risk factors. Nat Rev Rheumatol. 2015;11(11):64962. https://doi.org/10.1038/nrrheum.2015.91.

2. Perez-Ruiz F, Dalbeth N, Bardin T. A review of uric acid, crystal deposition disease, and gout. Adv Ther. 2015;32(1):31-41. https://doi.org/10.1007/s1232 5-014-0175-z.

3. Chou H, Chin TY, Peh WC. Dual-energy CT in gout - a review of current concepts and applications. J Med Radiat Sci. 2017;64(1):41-51. https://doi. org/10.1002/jmrs.223.

4. Pascual E, Sivera F, Andrés M. Synovial fluid analysis for crystals. Curr Opin Rheumatol. 2011;23(2):161-9. https://doi.org/10.1097/BOR.0b013e32 $8343 e 458$

5. Coupal TM, Mallinson Pl, Gershony SL, McLaughlin PD, Munk PL, Nicolaou S, et al. Getting the Most from your dual-energy scanner: recognizing, reducing, and eliminating artifacts. Am J Roentgenol. 2016;206(1):119-28. https://doi.org/10.2214/AJR.14.13901.

6. Glazebrook KN, Guimarães LS, Murthy NS, Black DF, Bongartz T, Manek NJ, et al. Identification of intraarticular and periarticular uric acid crystals with dual-energy CT: initial evaluation. Radiology. 2011;261(2):516-24. https://doi. org/10.1148/radiol.11102485.

7. Hu MG, Li SL, Lyu GR, Wang CF. Diagnostic value of high frequency ultrasound and dual-energy $C T$ for gouty arthritis. Chin J Med Imaging Technol. 2014;30(6):909-12.

8. Bongartz T, Glazebrook KN, Kavros SJ, Murthy NS, Merry SP, Franz WB, et al. Dual-energy $\mathrm{CT}$ for the diagnosis of gout: an accuracy and diagnostic yield study. Ann Rheum Dis. 2015;74(6):1072-7. https://doi.org/10.1136/a nnrheumdis-2013-205095.

9. Wu HX, Xue J, Ye L, Zhou QJ, Shi D, Xu RZ. The application of dual-energy computed tomography in the diagnosis of acute gouty arthritis. Clin Rheumatol. 2014;33(7):975-9. https://doi.org/10.1007/s10067-014-2606-2.

10. Neogi T, Jansen TLTA, Dalbeth N, Fransen J, Schumacher HR, Berendsen D, et al. 2015 gout classification criteria: an American College of Rheumatology/European league against rheumatism collaborative initiative. Ann Rheum Dis. 2015;74(10):1789-98. https://doi.org/10.1136/a nnrheumdis-2015-208237.

11. Gamala M, Jacobs JWG, Laar JM. The diagnostic performance of dual energy $C T$ for diagnosing gout: a systematic literature review and meta-analysis. Rheumatology (Oxford). 2019;58(12):2117-21. https://doi.org/10.1093/ rheumatology/kez180.

12. Zhang $B$, Yang $M$, Wang H. Diagnostic value of ultrasound versus dualenergy computed tomography in patients with different stages of acute gouty arthritis. Clin Rheumatol. 2020;39(5):1649-53. https://doi.org/10.1007/ s10067-020-05014-6.

13. Wang $Y$, Deng $X, X u Y$, Ji L, Zhang Z. Detection of uric acid crystal deposition by ultrasonography and dual-energy computed tomography a cross-sectional study in patients with clinically diagnosed gout. Medicine (Baltimore). 2018;97(42):e12834. https://doi.org/10.1097/MD. 0000000000012834.

14. Jia E, Zhu J, Huang W, Chen X, Li J. Dual-energy computed tomography has limited diagnostic sensitivity for short-term gout. Clin Rheumatol. 2018;37(3): 773-7. https://doi.org/10.1007/s10067-017-3753-z.

15. Bayat S, Aati O, Rech J, Sapsford M, Cavallaro A, Lell M, et al. Development of a dual-energy computed tomography scoring system for measurement of Urate deposition in gout. Arthritis Care Res (Hoboken). 2016;68(6):769-75. https://doi.org/10.1002/acr.22754.

16. Melzer R, Pauli C, Treumann T, Krauss B. Gout tophus detection-a comparison of dual-energy CT (DECT) and histology. Semin Arthritis Rheum. 2014;43(5):662-5. https://doi.org/10.1016/j.semarthrit.2013.11.002.

17. Roddy E, Doherty M. Epidemiology of gout. Arthritis Res Ther. 2010;12(6): 223. https://doi.org/10.1186/ar3199.

18. Dalbeth N, Kalluru R, Aati O, Horne A, Doyle AJ, McQueen FM. Tendon involvement in the feet of patients with gout: a dual-energy $C T$ study. Ann Rheum Dis. 2013;72(9):1545-8. https://doi.org/10.1136/a nnrheumdis-2012-202786.

19. Li X, Wang X, Yu Y, Liu B, Cai J, Xia L, et al. Detection of uric acid depositing in tophaceous gout using a new dual energy spectral CT technology. J Xray Sci Technol. 2014;22(4):541-9. https://doi.org/10.3233/XST-140444.

20. Monu JUV, Pope TL. Gout: a clinical and radiologic review. Radiol Clin N Am. 2004;42(1):169-84. https://doi.org/10.1016/50033-8389(03)00158-1.

21. Choi HK, Curhan G. Independent impact of gout on mortality and risk for coronary heart disease. Circulation. 2007;116(8):894-900. https://doi.org/1 0.1161/CIRCULATIONAHA.107.703389.

22. Lee SK, Jung J-Y, Jee W-H, Lee JJ, Park S-H. Combining non-contrast and dual-energy CT improves diagnosis of early gout. Eur Radiol. 2019;29(3): 1267-75. https://doi.org/10.1007/s00330-018-5716-4.

23. Diekhoff T, Kiefer T, Stroux A, Pilhofer I, Juran R, Mews J, et al. Detection and characterization of crystal suspensions using single-source dual-energy computed tomography: a phantom model of crystal arthropathies. Investig Radiol. 2015;50(4):255-60. https://doi.org/10.1097/RLI.0000000000000099.

24. Ogdie A, Taylor WJ, Weatherall M, Fransen J, Jansen TL, Neogi T, et al. Imaging modalities for the classification of gout: systematic literature review and meta-analysis. Ann Rheum Dis. 2015;74(10):1868-74. https://doi.org/1 0.1136/annrheumdis-2014-205431.

25. Filippou G, Pascart T, lagnocco A. Utility of ultrasound and dual energy $C T$ in crystal disease diagnosis and management. Curr Rheumatol Rep. 2020; 22(5):15. https://doi.org/10.1007/s11926-020-0890-1.

26. Dalbeth N, Nicolaou S, Baumgartner S, Hu J, Fung M, Choi HK. Presence of monosodium urate crystal deposition by dual-energy $C T$ in patients with gout treated with allopurinol. Ann Rheum Dis. 2018;77(3):364-70. https:// doi.org/10.1136/annrheumdis-2017-212046.

27. Urano W, Yamanaka H, Tsutani H, Nakajima H, Matsuda Y, Taniguchi A, et al. The inflammatory process in the mechanism of decreased serum uric acid concentrations during acute gouty arthritis. J Rheumatol. 2002;29(9):1950-3.

28. Dehlin M, Ekstrom EH, Petzold M, Stromberg U, Telg G, Jacobsson LT. Factors associated with initiation and persistence of urate-lowering therapy. Arthritis Res Ther. 2017;19(1):6. https://doi.org/10.1186/s13075-016-1211-y.

29. Dalbeth N, Billington K, Doyle A, Frampton C, Tan P, Aati O, et al. Effects of allopurinol dose escalation on bone Erosion and Urate volume in gout: a dual-energy computed tomography imaging study within a randomized, controlled trial. Arthritis Rheumatol. 2019;71(10):1739-46. https://doi.org/10.1 002/art.40929.

30. Ellmann H, Bayat S, Araujo E, Manger B, Kleyer A, Cavallaro A, et al. Effects of conventional uric acid-lowering therapy on monosodium Urate crystal deposits. Arthritis Rheumatol. 2020;72(1):150-6. https://doi. org/10.1002/art.41063.

31. Svensson $E$, Aurell $Y$, Jacobsson $L T H$, Landgren $A$, Sigurdardottir $V$, Dehlin $M$. Dual energy $C T$ findings in gout with rapid kilovoltage-switching source with gemstone scintillator detector. BMC Rheumatol. 2020;4(1):7. https://doi. org/10.1186/s41927-019-0104-5.

32. Dalbeth N, Clark B, Gregory K, Gamble G, Sheehan T, Doyle A, et al. Mechanisms of bone erosion in gout: a quantitative analysis using plain radiography and computed tomography. Ann Rheum Dis. 2009;68(8): 1290-5.

33. Megibow AJ, Kambadakone A, Ananthakrishnan L. Dual-energy computed tomography: image acquisition, processing, and workflow. Radiol Clin N Am. 2018;56(4):507-20. https://doi.org/10.1016/j.rcl.2018.03.001. 
34. Agostini A, Borgheresi A, Mari A, Floridi C, Bruno F, Carotti M, et al. Dualenergy CT: theoretical principles and clinical applications. Radiol Med. 2019; 124(12):1281-95. https://doi.org/10.1007/s11547-019-01107-8.

35. Chen H, Zhao L, Liu F, Chen S, Hu Z, Chen L, et al. Urate in fingernail represents the deposition of urate burden in gout patients. Sci Rep. 2020; 10(1):15575. https://doi.org/10.1038/s41598-020-72505-6.

36. Rajiah P, Parakh A, Kay F, Baruah D, Kambadakone AR, Leng S. Update on multienergy CT: physics, principles, and applications. Radiographics. 2020; 40(5):1284-308. https://doi.org/10.1148/rg.2020200038.

37. Foley WD, Shuman WP, Siegel MJ, Sahani DV, Boll DT, Bolus DN, et al. White paper of the Society of Computed Body Tomography and Magnetic Resonance on dual-energy $C T$, part 2: radiation dose and iodine sensitivity. J Comput Assist Tomogr. 2016;40(6):846-50. https://doi.org/10.1097/RCT. 0000000000000539 .

\section{Publisher's Note}

Springer Nature remains neutral with regard to jurisdictional claims in published maps and institutional affiliations.

Ready to submit your research? Choose BMC and benefit from:

- fast, convenient online submission

- thorough peer review by experienced researchers in your field

- rapid publication on acceptance

- support for research data, including large and complex data types

- gold Open Access which fosters wider collaboration and increased citations

- maximum visibility for your research: over $100 \mathrm{M}$ website views per year

At BMC, research is always in progress.

Learn more biomedcentral.com/submissions 